\title{
Medium response to jets in heavy ion collisions
}

\author{
Yasuki Tachibana ${ }^{1,2, \star}$ \\ ${ }^{1}$ Institute of Particle Physics and Key Laboratory of Quark and Lepton Physics (MOE), Central China Normal \\ University, Wuhan, Hubei 430079, China \\ ${ }^{2}$ Department of Physics and Astronomy, Wayne State University, Detroit, Michigan 48201, USA
}

\begin{abstract}
A short overview on recent progress in studies of medium response to jet quenching in heavy ion collisions is presented. We show the typical features of medium response and give comment on their connection to jet observables by introducing the work done by the author and collaborators as an example.
\end{abstract}

\section{Introduction}

The deconfined state of quarks and gluons, so-called quark-gluon plasma (QGP), has been created and explored experimentally in heavy ion collisions at the Relativistic Heavy Ion Collider (RHIC) and the Large Hadron Collider (LHC) [1]. In particular, the observation of the large anisotropic flow patterns indicates the collective behavior of the bulk QGP, which implies the strong interactions among the constituents. Various relativistic hydrodynamic models have been developed and very successful in explaining the space-time evolution of the bulk QGP [2-10]. As another remarkable feature of the strongly coupled QGP, jet quenching, namely the large energy loss of jets, has also been revealed in heavy ion collisions $[11,12]$. The jets are collimated sprays of particles resulting from the QCD showering of high- $p_{\mathrm{T}}$ partons produced in early stage hard scatterings. Under the existence of the QGP medium, partons in jets collide and exchange the energy and momentum with the medium constituents via the strong interactions during the penetration in the medium. In addition, the successive collisions inside the medium cause bremsstrahlung in QCD and induce additional partonic showering in the jets (medium-induced radiation). Through the collisions and medium-induced radiations, the structure of the jets is significantly modified, and their modification patterns provide the information of the interactions between jets and QGP medium.

Medium response, the effect of the wake generated by the jet propagation in the medium, is another possible contribution to the medium modification of the jet structures and has recently been focused [13-19]. The energy and momentum deposited by the jets are supposed to excite the medium, and induce flows following the jets. The jet-induced flow carries the deposited energy and momentum and enhances the hadron emission from the medium around the jet axis direction. Since the medium response happens only together with the jet propagation, these enhanced hadrons correlated with the jet propagation cannot be subtracted with background and detected with the fragments from the energetic jet shower.

^e-mail: yasuki.tachibana@wayne.edu 


\section{Motivations}

(a)

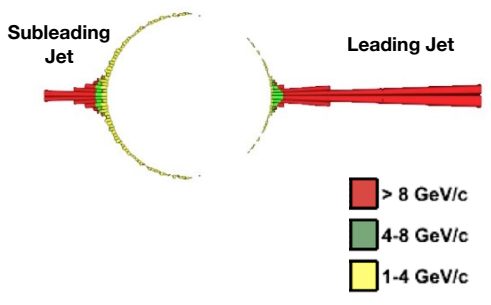

(b)

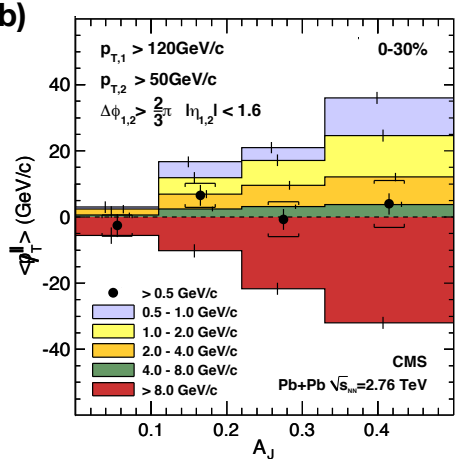

Figure 1. (a) Azimuthal angle distribution of transverse momentum in the transverse plane in a dijet event at LHC observed by CMS Collaboration. Each color in histgrams represents the contribution in a transversemomentum range. Figure is adapted from the slides of Ref. [20]. (b) Missing transverse momentum $\left\langle p_{\mathrm{T}}^{\|}\right\rangle=$ $\left\langle-\sum_{i} p_{\mathrm{T}}^{i} \cos \left(\phi_{i}-\phi_{\text {Leading Jet }}\right)\right\rangle$ as a function of dijet asymmetry ratio $A_{J}=\frac{p_{\mathrm{T}, 1}-p_{\mathrm{T}, 2}}{p_{\mathrm{T}, 1}+p_{\mathrm{T}, 2}}$ measured for dijet events in $\mathrm{PbPb}$ collisions at LHC by CMS Collaboration [21]. The solid black circles show the total $\left\langle p_{\mathrm{T}}^{\|}\right\rangle$and each color in histograms represents the contribution in a transverse-momentum range. Figure is adapted from Ref. [21].

Detailed measurements of fully reconstructed jets and their correlated particles provide us information on a lot of physics over a wide range of energy-momentum scales involved in jet quenching phenomenon. For example, CMS Collaboration observed dijet events with large $p_{\mathrm{T}}^{\text {jet }}$-imbalance in $\mathrm{PbPb}$ collisions at the LHC [21]. Figure 1 (a) is the azimuthal angle-distribution of the transverse momentum in a dijet event. One can see two peaks; the taller peak on the right side for the leading jet and the shorter peak on the left side for the subleading jet. As a consequence of the jet quenching effect, the transverse momentum of the leading jet $p_{\mathrm{T}, 1}$ and the subleading jet $p_{\mathrm{T}, 2}$ are uneven and the large asymmetry $\left(p_{\mathrm{T}, 1}>p_{\mathrm{T}, 2}\right)$ can be seen. However, at large angles from the jet axes, a huge enhancement of low- $p_{\mathrm{T}}$ particles is observed together with the jets. When the contribution of these low- $p_{\mathrm{T}}$ particles is taken into account, the whole transverse momentum in a dijet event turns out to be balanced. This transverse-momentum balance is confirmed for any asymmetric dijet events [Fig. 1 (b)] (further details can be found in Ref. [21]), and shows evidence of momentum conservation in dijet events in heavy ion collisions. The enhanced low- $p_{\mathrm{T}}$ particles originate from the energy and momentum of the jets redistributed through the jet quenching effect, and can naturally be considered as a consequence of the medium response because their transverse momenta are sufficiently low [15, 22, 23]. Accordingly, to study the full picture of jet quenching phenomenon it is necessary to consider the effect of medium response.

Medium response effect may give a manifestation of the QGP's fluidity associated with jets and information about the bulk QGP properties, e.g. sound velocity, and transport coefficients. For the QGP fluid, the energetic partons in jets can be considered fast-moving sources depositing the energy and momentum, which are supposed to excite the conical shockwave, so-called Mach cone, as a hydrodynamic medium response [24-28]. The shockwave front propagates toward a specific angle from the direction of the moving source which is determined by the sound velocity of the fluid. The shape of the Mach cone can also be affected by the finite viscosities of the QGP fluid, e.g., the shockwave front can be smeared by the shear viscosity [29-36]. Furthermore, medium response involves the 
QGP transport properties related to how part of the jet energy and momentum relaxes into the hydro mode in the QGP medium. This relaxation process has not been studied well but is very important to define the scale separation between the hard jet part and the soft fluid part, and to obtain the space-time profile of the energy-momentum deposition into the QGP fluid [29, 37, 38].

\section{Modelings for medium response in recent studies}

Recently, various models for numerical simulation studies with inclusion of the medium response effect have been developed and used to show the contribution of the effect on the jet sub-structure observables. Some event-by-event models for in-medium jet evolution include the medium response as recoil partons. In this approach, when they consider a two-particle (t-channel) scattering between a jet parton and a medium constituent, a sample of parton from the medium is taken with the thermal distribution. Then after the scattering, the scattered parton from the thermal medium is called the recoil parton and tracked as part of the jet partons. Jet Evolution With Energy Loss (JEWEL) [18, 19, 39, 40], the jet-quenching Monte-Carlo code based on the BDMPS-Z formalism [41-45], has the option to include the recoil partons traveling freely in the medium without any further interaction. In the recent version of Linear Boltzmann Transport (LBT) model [13, 46-48], the recoil partons is implemented together with the in-medium partonic radiation based on the higher-twist formalism [49, 50]. In LBT model, the recoil partons also take part in the interactions with the medium constituents, and the appearance of the Mach cone-like structure that can also be seen in jet studies with Boltzmann Approach of MultiParton Scatterings (BAMPS) model [32, 33] or A Multi-Phase Transport (AMPT) model [23] is shown.

When part of the jet energy and momentum is thermalized and deposited into the QGP fluid, the dynamics of the medium response can be described by relativistic hydrodynamics. To study the hydrodynamic medium response, the hydrodynamic equation with source term,

$$
\partial_{\mu} T_{\text {fluid }}^{\mu v}(x)=J^{v}(x)
$$

is commonly used $[16,17,22,29-31,51-62]$. Here, $T_{\text {fluid }}^{\mu \nu}(x)$ is the energy-momentum tensor for the medium fluid, and $J^{v}(x)$ is the source term representing the space-time profile of the energy and momentum deposited by jets. In the recent studies of full jet shower with hydrodynamic medium response, Eq. (1) is solved while being coupled to jet transport equations; the source term in Eq. (1) is calculated from the solutions of the jet transport equations based on the energy-momentum conservation for the combined system of the jets and the QGP fluid. It should be noted that the hydrodynamic simulation with the jet source term must be performed in full $(3+1)$-dimensional space time for a complete theoretical treatment, since the jet-induced flow, e.g. Mach cone, always has a three-dimensional structure and violates the boost invariance that the fluid profile is supposed to have as an approximate symmetry around midrapidity. The hadrons from the medium fluid with medium response are obtained by using Copper-Frye formula [63], which is most commonly used in hydrodynamic models for heavy ion collisions. After the background subtraction, the hadrons from the medium fluid are summed to the final-state particles calculated from the jet transport equation. Then, the jet including the contribution of the hydrodynamic medium response is obtained. An example of this approach is the model used in Ref. [16] (we refer it as Jet Shower Transport + Hydro model below). In this model, it is assumed that all partons scattered by the jet partons instantaneously rethermalize in the medium fluid. As another example, Coupled LBT Hydro model [17] is a full event-by-event model employing LBT Model for the jet evolution calculation. In this model, they assume that all the jet partons in LBT simulation with energy below a certain cutoff energy are completely absorbed in the medium fluid, and deposit their energy and momentum. In this framework, the contribution of both hydrodynamic 
medium response and recoil partons can be handled. Incidentally, most recently, it is suggested to use Eq. (1) not only for the medium response to jets but also for the early space-time evolution of the QGP fluid that gradually forms while receiving the energy and momentum deposited by the matters in the pre-thermalized (or pre-hydronized) state [64, 65].

In addition, also the latest version of Hybrid Strong/Weak Coupling model $[15,66]$ contains a simplified description for the medium response. The lost energy and momentum of the jet calculated by $\mathcal{N}=4$ supersymmetric Yang-Mills theory [67] are stored into a boost-invariant Bjorken fluid as a perturbation, and then the hadrons enhanced by the perturbation are obtained by using the linear expansion of Cooper Frye formula. In this approach, the space-time evolution of the jet-induced flow is not described.

\section{Full jet study with Jet Shower Transport + Hydro model}

Here we briefly introduce the full jet study by Jet Shower Transport + Hydro model [16] and show the typical features of medium response contribution in jet observables. Some calculations with other models show different results but mostly they are the consequences of the competition with the effects other than the medium response.

\subsection{Jet Shower Transport + Hydro model}

In Jet Shower Transport + Hydro model, the relativistic hydrodynamic equations with source term (1) is numerically solved in $(3+1)$ dimensional space-time to study the space-time evolution of the medium with energy-momentum deposition from the jet. Neglecting the small viscosity of the medium, the medium is modeled as an ideal fluid here. The initial profile of the medium fluid for central $\mathrm{PbPb}$ collisions at $2.76 \mathrm{~A} \mathrm{TeV}$ is generated by the optical Glauber model with the impact parameter $b=0$. The jet shower evolution is described in terms of the energy and transverse momentum distributions of the partons in the jet shower, $f_{i}\left(\omega_{i}, k_{i \perp}^{2}, t\right)=d N_{i}\left(\omega_{i}, k_{i \perp}^{2}, t\right) / d \omega_{i} d k_{i \perp}^{2}$, by solving the transport equation [68],

$$
\frac{d f_{i}}{d t}=\left(\hat{e}_{i} \frac{\partial f_{i}}{\partial \omega_{i}}+\frac{1}{4} \hat{q}_{i} \nabla_{k_{i \perp}}^{2} f_{i}\right)+\sum_{j} \int d \omega_{j} d k_{j \perp}^{2}\left[\frac{d \tilde{\Gamma}_{j \rightarrow i}\left(\omega_{i}, k_{i \perp}^{2} \mid \omega_{j}, k_{j \perp}^{2}\right)}{d \omega_{i} d k_{i \perp}^{2} d t} f_{j}-\frac{d \tilde{\Gamma}_{i \rightarrow j}\left(\omega_{j}, k_{j \perp}^{2} \mid \omega_{i}, k_{i \perp}^{2}\right)}{d \omega_{j} d k_{j \perp}^{2} d t} f_{i}\right] .
$$

The first two terms describe the energy-momentum changes via the scatterings with the medium constituents in the longitudinal direction (collisional energy loss), and in the transverse direction (transverse momentum broadening), respectively. The last two terms account for the medium-induced radiations. In this framework, all the strengths of the medium effects are determined solely by the jet quenching parameter for quarks $\hat{q}_{q}$ (see Ref. [68] for the details of the model for the jet shower evolution, and Ref. [16] for the choice of the parameters).

Assuming that the energy and momentum transferred to the medium partons from the jet are quickly thermalized, the source term $J^{v}(x)$ in Eq. (1) is constructed by using the solution of Eq. (2) as follows:

$$
J^{v}(x)=-\sum_{i} \int \frac{d \omega_{i} d k_{i \perp}^{2} d \phi_{i}}{2 \pi} k_{i}^{v}\left[\left(\hat{e}_{i} \frac{\partial}{\partial \omega_{i}}+\frac{1}{4} \hat{q}_{i} \nabla_{k_{\perp}}^{2}\right) f_{i}\left(\omega_{i}, k_{i \perp}^{2}, t\right)\right] \delta^{(3)}\left(\boldsymbol{x}-\boldsymbol{x}_{0}^{\mathrm{jet}}-\frac{\boldsymbol{k}_{i}}{\omega_{i}} t\right) .
$$

Here, the jets are generated by the Glauber model+PyтніA $[69,70]$, and assumed to be created in the transverse plane at $\eta_{\mathrm{s}}=0$. The initial proper time of the hydrodynamic medium evolution and jetmedium interaction is set as $\tau_{0}=0.6 \mathrm{fm} / c$. Hereafter we refer to the contribution of particles in jet shower described by Eq. (2) as shower part, and to that of particles coming from the hydrodynamic medium response as hydro part. 


\subsection{Results}
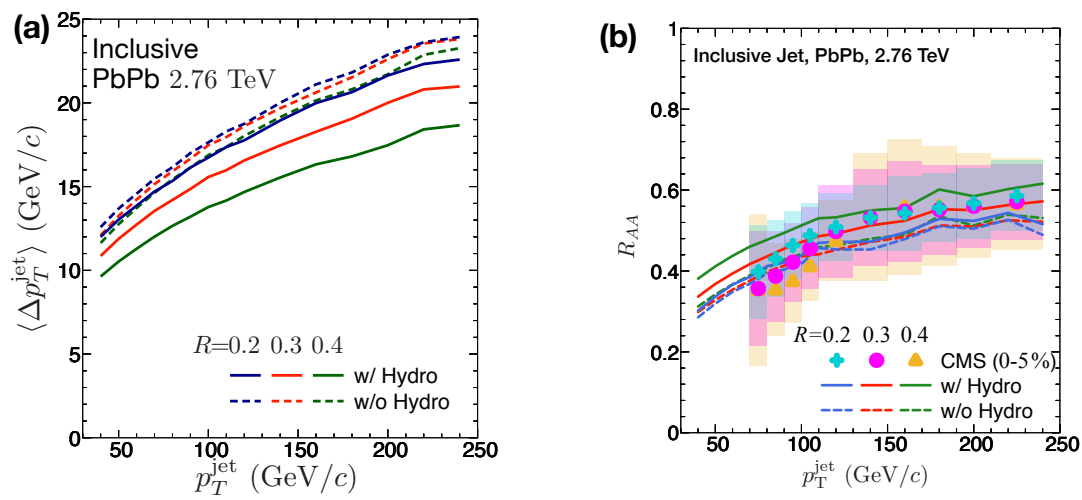

Figure 2. (a) Total $p_{\mathrm{T}}^{\text {jet }}$ loss as a function of initial $p_{\mathrm{T}}^{\text {jet }}$, and (b) nuclear modification factor for inclusive jets in central $\mathrm{PbPb}$ collisions at $2.76 \mathrm{~A} \mathrm{TeV}$ with the jet-cone sizes $R=0.2,0.3$, and 0.4 [16]. The solid lines show the results for jets with hydro part, and the dashed lines shows those for jets without hydro part. Experimental data are measured by the CMS Collaboration [71]. The colored shaded boxes denote the systematic uncertainties.

The jets in the final state are contributed from two parts: the shower part and the hydro part. The shower part loses energy due to three types of mechanism:

1. The collisional energy loss reduces the energy of all the jet shower partons.

2. The transverse momentum broadening tends to kick the jet shower partons out of the jet cone.

3. Medium-induced radiated partons outside the jet cone carry away the energy from the jet shower.

On the other hand, some of the particles from the hydro part carring the energy deposited by the jets via the mechanisms 1 and 2 are emitted inside the jet cone and detected as part of the jet. In this way, the lost energy from the shower part is partially recovered by the hydro part. To see this effect, the mean value of the total transverse momentum loss $\left\langle\Delta p_{\mathrm{T} \text {,init }}^{\text {jet }}\right\rangle=\left\langle p_{\mathrm{T} \text {,init }}^{\text {jet }}-p_{\mathrm{T} \text {,final }}^{\text {jet }}\right\rangle$ for inclusive jets as a function of $p_{\mathrm{T} \text {,init }}^{\text {jet }}$ is shown in Fig. 2 (a). One can see the feature that the total $p_{\mathrm{T}}^{\text {jet }}$ loss with the hydro part is smaller than that without the hydro part due to the partial energy-momentum recovery mentioned above. Also, the jet cone size dependence becomes stronger when the hydro part is included. This is because more energy and momentum carried by the hydro part are recovered by the increasing of jet cone size. These effects of the hydro part can be seen also in the nuclear modification factor for single inclusive jet spectrum $R_{\mathrm{AA}}=\left(1 /\left\langle N_{\text {coll }}\right\rangle\right)\left(d^{2} N_{\text {jet }}^{\mathrm{AA}} / d \eta_{p} d p_{\mathrm{T}}^{\mathrm{jet}}\right) /\left(d^{2} N_{\text {jet }}^{\mathrm{pp}} / d \eta_{p} d p_{\mathrm{T}}^{\mathrm{jet}}\right)$ shown in Fig. $2(\mathrm{~b})$.

Figure 3 shows the jet shape function for leading and subleading jets in dijet events. The jet shape function is defined as

$$
\rho(r)=\frac{1}{N_{\text {jet }}} \sum_{\text {jet }}\left[\frac{1}{p_{T}^{\mathrm{jet}}} \frac{\sum_{\mathrm{trk} \in(\mathrm{r}-\delta \mathrm{r} / 2, \mathrm{r}+\delta \mathrm{r} / 2)} p_{T}^{\mathrm{trk}}}{\delta r}\right],
$$

where $r=\sqrt{\left(\eta_{\mathrm{p}}-\eta_{\mathrm{jet}}\right)^{2}+\left(\phi-\phi_{\mathrm{jet}}\right)^{2}}$. In the small- $r$ region, the hydro part does not affect much the jet shape. However, the contribution of the hydro part becomes larger by increasing the value of $r$, 

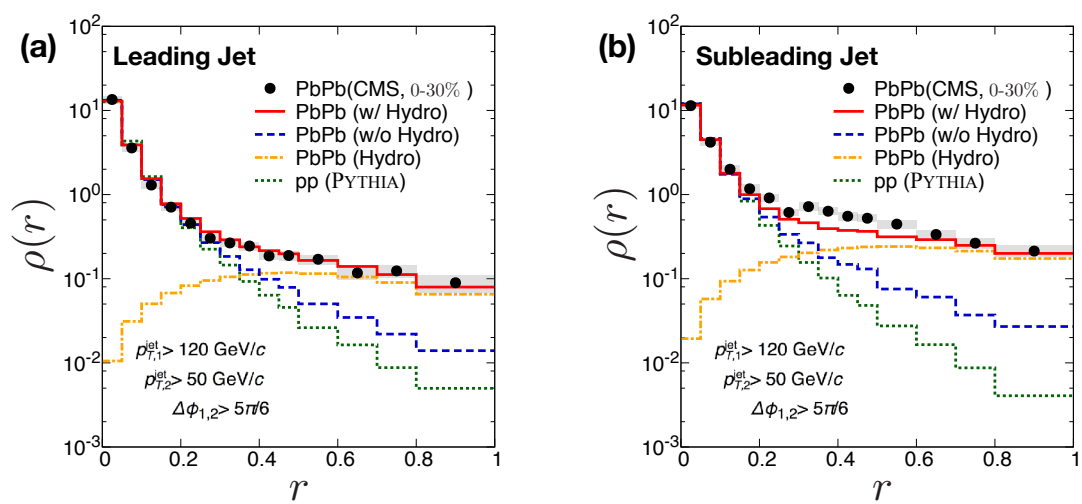

Figure 3. Jet shape functions for (a) leading and (b) subleading jet in dijet events at $2.76 \mathrm{~A} \mathrm{TeV} \mathrm{[16].} \mathrm{The} \mathrm{dijet}$ events are selected with the leading jet, $p_{T, 1}^{\text {jet }}>120 \mathrm{GeV} / c$, the subleading jet, $p_{T, 2}^{\text {jet }}>50 \mathrm{GeV} / c$, and the relative azimuth angle between the leading and subleading jets, $\Delta \phi_{1,2}>5 \pi / 6$. The solid lines and dashed lines show the results for jets with and without hydro part in central $\mathrm{PbPb}$ collisions, respectively. The dashed dotted lines are the hydro part contribution in the jets with hydro part. The dotted lines are the results for $\mathrm{p}+\mathrm{p}$ collisions obtained by PYтнIA. Experimental data are measured by the CMS Collaboration [71] (the shaded boxes indicate the systematic uncertainties).

and finally in the very large- $r$ region $(r \gtrsim 0.5)$, the contribution of the hydro part dominates the jet shape function. The result including the significant broadening by the hydro part provides a good description of the experimental data from the CMS collaboration [71] in the whole region $(0 \leq r \leq 1)$, and indicates that jet-induced flow can spread quite widely around the jet axis. This means that the particles emitted at large angle regions with respect to the jet axis direction provide a good opportunity to study the medium response.

\section{Conclusion, comments and outlook}

Medium response to jets in heavy ion collisions appears as the excitation of the QGP medium caused by the jet energy-momentum deposition. The jet-correlated hadrons emitted by the excited medium can be detected as part of the jet and affect the energy and structures of jets in the final state. In the full jet study with Jet Shower Transport + Hydro model, the sizable contribution of the energymomentum recovered by the medium response has been seen. The hadrons from the medium response are supposed to have small $p_{\mathrm{T}}$ compared with the hadrons fragmented from the hard intrinsic jet shower, and modify the hadron $p_{\mathrm{T}}$ distribution in jets. In Ref. [17], the $\gamma$-triggered jet fragmentation function and $\gamma$-hadron correlation as a function of $\xi=-\ln p_{\mathrm{T}}^{h} / p_{\mathrm{T}}^{\gamma}$ are calculated at RHIC energy by Coupled LBT Hydro model, and the significant enhancement of soft (large- $\xi$ ) hadrons due to the jet-induced medium excitation is shown. Also in a study with JEWEL [19], they show that the soft hadrons from the recoil partons modify the $z_{\mathrm{g}}$-distribution in jets. The energy and momentum deposited in the medium are redistributed to the wide-angle region around the jet axis direction by the medium response. As a result, increase of the jet-cone size dependence in jet energy loss and the strong enhancement of jet shape at large- $r$ have been seen in the results from Jet Shower Transport + Hydro model. The large number of soft particles at large angles from the jet axis due to the medium response effect is shown in the calculations of missing transverse momentum from various models such as hydrodynamics with source term [22], Hybrid Strong/Weak Coupling model [15], and AMPT [23] as well. 
Medium response may provide new approaches to extract the QGP's transport properties from jet events. The shear viscosity dependence of the jet-induced flow pattern and the resulting jet-hadron correlation have been studied in several approaches based on linearized hydrodynamics [29-31, 34$36]$, or BSMPS model calculation [32, 33]. For further detailed study to connect them to realistic observables in heavy ion collisions, full $(3+1)$-dimensional viscous hydrodynamic model simulations would need to be done. While we have mostly focused on the dynamics of the deposited energy and momentum after their relaxation into the hydro mode, the detailed mechanism of the relaxation process is not fully understood but needed to be considered when obtaining the finite structure of the source term in Eq. (1). It is one of the essential problems of the jet study in heavy ion collisions, which defines the separation between the hard intrinsic jet scale and the soft medium fluid scale.

Thanks to a wealth of the experimental data and their sophisticated analyses, observables containing rich information of jet structures have become available. Medium response is an essential ingredient for an integrated theoretical framework for the jet study which should be developed for the interpretation of the experimental data from various aspects in order to achieve a comprehensive understanding of the jet quenching phenomenon.

\section{Acknowledgements}

The author would like to thank N.-B. Chang, T. Hirano, G.-Y. Qin, and X.-N. Wang for discussions on this topic. The author was supported in part by the Natural Science Foundation of China (NSFC) under Grants No. 11375072 and No. 11405066, by the Chinese Ministery of Science and Technology under Grant No. 2014DFG02050, and by the Major State Basic Research Development Program in China under Grant No. 2014CB845404.

\section{References}

[1] K. Yagi, T. Hatsuda, Y. Miake, Camb. Monogr. Part. Phys. Nucl. Phys. Cosmol. 23, 1 (2005)

[2] U.W. Heinz, P.F. Kolb, Nucl. Phys. A702, 269 (2002)

[3] T.D. Lee, Nucl. Phys. A750, 1 (2005)

[4] M. Gyulassy, L. McLerran, Nucl. Phys. A750, 30 (2005)

[5] E.V. Shuryak, Nucl. Phys. A750, 64 (2005)

[6] T. Hirano, M. Gyulassy, Nucl. Phys. A769, 71 (2006)

[7] B. Schenke, S. Jeon, C. Gale, Phys. Rev. Lett. 106, 042301 (2011)

[8] Z. Qiu, U.W. Heinz, Phys. Rev. C84, 024911 (2011)

[9] Z. Qiu, C. Shen, U. Heinz, Phys. Lett. B707, 151 (2012)

[10] C. Gale, S. Jeon, B. Schenke, P. Tribedy, R. Venugopalan, Phys. Rev. Lett. 110, 012302 (2013)

[11] J.P. Blaizot, Y. Mehtar-Tani, Int. J. Mod. Phys. E24, 1530012 (2015)

[12] G.Y. Qin, X.N. Wang, Int. J. Mod. Phys. E24, 1530014 (2015)

[13] X.N. Wang, Y. Zhu, Nucl. Phys. A932, 447 (2014)

[14] Y. He, T. Luo, X.N. Wang, Y. Zhu, Phys. Rev. C91, 054908 (2015)

[15] J. Casalderrey-Solana, D. Gulhan, G. Milhano, D. Pablos, K. Rajagopal, JHEP 03, 135 (2017)

[16] Y. Tachibana, N.B. Chang, G.Y. Qin, Phys. Rev. C95, 044909 (2017)

[17] W. Chen, S. Cao, T. Luo, L.G. Pang, X.N. Wang (2017)

[18] R. Kunnawalkam Elayavalli, K.C. Zapp, JHEP 07, 141 (2017)

[19] J.G. Milhano, U.A. Wiedemann, K.C. Zapp, arXiv : 1707.04142

[20] C. Roland, J.Phys. G38, 124020 (2011) 
[21] S. Chatrchyan et al. (CMS Collaboration), Phys. Rev. C84, 024906 (2011)

[22] Y. Tachibana, T. Hirano, Phys. Rev. C90, 021902 (2014)

[23] Z. Gao, G.L. Ma, G.Y. Qin, H.Z. Zhang, arXiv: 1612.02548

[24] H. Stoecker, Nucl. Phys. A750, 121 (2005)

[25] J. Casalderrey-Solana, E.V. Shuryak, D. Teaney, J.Phys.Conf.Ser. 27, 22 (2005)

[26] J. Ruppert, B. Muller, Phys. Lett. B618, 123 (2005)

[27] L.M. Satarov, H. Stoecker, I.N. Mishustin, Phys. Lett. B627, 64 (2005)

[28] T. Renk, J. Ruppert, Phys. Rev. C73, 011901 (2006)

[29] R.B. Neufeld, Phys. Rev. C79, 054909 (2009)

[30] R.B. Neufeld, T. Renk, Phys. Rev. C82, 044903 (2010)

[31] R.B. Neufeld, I. Vitev, Phys. Rev. C86, 024905 (2012)

[32] I. Bouras, A. El, O. Fochler, H. Niemi, Z. Xu et al., Phys. Lett. B710, 641 (2012)

[33] I. Bouras, B. Betz, Z. Xu, C. Greiner, Phys. Rev. C90, 024904 (2014)

[34] A. Ayala, J.D. Castaño-Yepes, I. Dominguez, M.E. Tejeda-Yeomans, Phys. Rev. C92, 024910 (2015)

[35] A. Ayala, I. Dominguez, J. Jalilian-Marian, M.E. Tejeda-Yeomans, Phys. Rev. C94, 024913 (2016)

[36] L. Yan, S. Jeon, C. Gale, arXiv: 1707.09519

[37] T. Renk, Phys. Rev. C88, 044905 (2013)

[38] E. Iancu, B. Wu, JHEP 10, 155 (2015)

[39] K.C. Zapp, F. Krauss, U.A. Wiedemann, JHEP 1303, 080 (2013)

[40] K.C. Zapp, Eur. Phys. J. C74, 2762 (2014)

[41] R. Baier, Y.L. Dokshitzer, A.H. Mueller, S. Peigne, D. Schiff, Nucl. Phys. B483, 291 (1997)

[42] B.G. Zakharov, JETP Lett. 63, 952 (1996)

[43] R. Baier, Y.L. Dokshitzer, A.H. Mueller, S. Peigne, D. Schiff, Nucl. Phys. B484, 265 (1997)

[44] B.G. Zakharov, JETP Lett. 65, 615 (1997)

[45] R. Baier, Y.L. Dokshitzer, A.H. Mueller, D. Schiff, Nucl. Phys. B531, 403 (1998)

[46] T. Luo, Y. He, E. Wang, X.N. Wang, Nucl. Phys. A956, 585 (2016)

[47] S. Cao, T. Luo, G.Y. Qin, X.N. Wang, Phys. Rev. C94, 014909 (2016)

[48] S. Cao, T. Luo, G.Y. Qin, X.N. Wang, arXiv: 1703.00822

[49] X.N. Wang, X.f. Guo, Nucl. Phys. A696, 788 (2001)

[50] A. Majumder, Phys. Rev. D85, 014023 (2012)

[51] A.K. Chaudhuri, U. Heinz, Phys. Rev. Lett. 97, 062301 (2006)

[52] A.K. Chaudhuri, Phys. Rev. C75, 057902 (2007)

[53] A.K. Chaudhuri, Phys. Rev. C77, 027901 (2008)

[54] R.B. Neufeld, B. Muller, J. Ruppert, Phys. Rev. C78, 041901 (2008)

[55] R.B. Neufeld, Phys. Rev. D78, 085015 (2008)

[56] R.B. Neufeld, B. Muller, Phys. Rev. Lett. 103, 042301 (2009)

[57] G.Y. Qin, A. Majumder, H. Song, U. Heinz, Phys. Rev. Lett. 103, 152303 (2009)

[58] B. Betz, J. Noronha, G. Torrieri, M. Gyulassy, D.H. Rischke, Phys. Rev. Lett. 105, 222301 (2010)

[59] R.P.G. Andrade, J. Noronha, G.S. Denicol, Phys. Rev. C90, 024914 (2014)

[60] S. Floerchinger, K.C. Zapp, Eur. Phys. J. C74, 3189 (2014)

[61] M. Schulc, B. Tomášik, Phys. Rev. C90, 064910 (2014) 
[62] Y. Tachibana, T. Hirano, Phys. Rev. C93, 054907 (2016)

[63] F. Cooper, G. Frye, Phys. Rev. D10, 186 (1974)

[64] M. Okai, K. Kawaguchi, Y. Tachibana, T. Hirano, Phys. Rev. C95, 054914 (2017)

[65] C. Shen, B. Schenke, arXiv: 1710.00881

[66] Z. Hulcher, D. Pablos, K. Rajagopal, arXiv: 1707.05245

[67] P.M. Chesler, K. Rajagopal, Phys. Rev. D90, 025033 (2014)

[68] N.B. Chang, G.Y. Qin, Phys. Rev. C94, 024902 (2016)

[69] M.L. Miller, K. Reygers, S.J. Sanders, P. Steinberg, Ann. Rev. Nucl. Part. Sci. 57, 205 (2007)

[70] T. Sjostrand, S. Mrenna, P.Z. Skands, Comput. Phys. Commun. 178, 852 (2008)

[71] V. Khachatryan et al. (CMS Collaboration), Phys. Rev. C96, 015202 (2017) 